\title{
Effect of Hypoxia on the Stability of Microcirculation Parameters in the Temporal Head Regions of Humans
}

\author{
L. V. Mezentseva ${ }^{a, *}$, E. N. Dudnik ${ }^{b}$, and E. V. Nikenina ${ }^{a}$ \\ ${ }^{a}$ Anokhin Research Institute of Normal Physiology, Moscow, 125315 Russia \\ ${ }^{b}$ Sechenov First Moscow State Medical University, Moscow, 119435 Russia \\ *e-mail: l.v.mezentseva@mail.ru \\ Received December 11, 2020; revised March 2, 2021; accepted April 30, 2021
}

\begin{abstract}
A new computational and experimental method for assessing the stability of oscillatory processes occurring in the microcirculation (MCR) system is proposed. The technique is based on the analysis of the stability of a differential equations system describing fluctuations in the MCR parameters of blood in symmetrical temporal head regions of humans. Using this technique, we have investigated the change in the stability of MCR parameters of the symmetrical temporal head regions under hypoxic conditions. The study has involved 10 healthy male volunteers (18-19 years). The state of short-term hypoxia has been induced using a normobaric ReOxy Cardio device (S.A. Aimediq, Luxembourg) certified in the Russian Federation for obtaining hypoxic and hyperoxic gas mixtures based on biofeedback with a built-in pulse oximetric sensor (Masimo Rad-5, United States). Synchronous measurements of MCR parameters of symmetrical temporal head regions at the basal state and immediately after short-term hypoxic exposure have been carried out by the method of laser Doppler flowmetry (LDF) using a two-channel LAKK-02 device (LAZMA Research and Development enterprise, Russia). It has been shown that short-term hypoxic effects change the numerical values of the stability parameters in all subjects within the boundaries of stability levels. However, the response to hypoxic exposure has been very individual in different subjects. The proposed method allows for personalized assessments of the fitness level and resistance to hypoxic influences in different subject groups.
\end{abstract}

Keywords: hypoxia, microcirculation, stability, laser Doppler flowmetry

DOI: $10.1134 / \mathrm{S} 0362119722010108$

Currently, a wealth of experimental data has been gained empirically, demonstrating the quantitative characteristics of perfusion parameters and vascular tone components in different organs and tissues of animals and humans [1-6]. The researches discovered the specificity of microcirculation control in paired organs associated with functional asymmetry $[1,2]$. Significant correlations were found between the microcirculation (MCR) parameters of symmetrical observation sides both at rest [1] and under external influences [2]. However, available empirical data on the studied processes do not explain the systemic regulatory mechanisms underlying these phenomena. In order to do this, the researches require a mathematical model of studied phenomenon, rather than fragmentary experimental data. The present computational and experimental study aims to mathematically analyze the fluctuations of MCR parameters in symmetrical head regions of humans.

The purpose of this study was to compose a mathematical model, allowing to assess the stability of MCR system under external influences. Short-term hypoxia was used as the external influence.

\section{METHODS}

MCR parameters were synchronously measured in 10 healthy male volunteers aged $18-19$ years using laser Doppler flowmetry (LDF). The tests were conducted with a two-channel LAKK-02 device (LAZMA Research and Development enterprise, Russia). LDF signal transducers were located on the outer surface of temporal head regions symmetrically, $1 \mathrm{~cm}$ laterally from the hairline. Short-term hypoxia was modeled according to the hypoxic test on ReOxy Cardio device (S.A. Aimediq, Luxembourg) with a built-in pulse oximetric sensor (Masimo Rad-5, United States). Adherent mouth and nose mask was fixed on the subject's face; finger was placed in the pulse oximeter; then the subjects were asked to breath tidally through the mask up to $10 \mathrm{~min}$. The test was performed at rest and in a sitting position with the arterial oxygen saturation $\left(\mathrm{SpO}_{2}\right)$ and heart rate $(\mathrm{HR}$, $\mathrm{bpm}$ ) monitored throughout the test and the individual values of minimal $\mathrm{SpO}_{2}$ and maximal $\mathrm{HR}$ were automatically registered. The oxygen concentration in the inhaled air was $11 \%$. The time of $\mathrm{SpO}_{2}$ reduction from the baseline (96-99\%) to $80 \%$ (desaturation 
time in seconds) was registered during the hypoxic test. The cessation criteria for hypoxic phase of the test were $\mathrm{SpO}_{2}$ reduction to $80 \%$ and below or after $9 \mathrm{~min}$ of breathing with hypoxic gas mixture. Integrated electronic stopwatch tracked the time automatically. Prominent tachycardia, namely a resistant increase in the HR by $30 \%$ and above from the initial level, was an additional criterion for hypoxic phase cessation. MCR parameters were recorded for each subject in the baseline condition before the test and immediately after the exposure to hypoxia. LDP signal was registered at a frequency of $20 \mathrm{~Hz}$ for $3 \mathrm{~min}$ with the counting interval of $\Delta t[i]=0.05 \mathrm{~s}$, resulting in over 3000 points in the corresponding periodogram. For further analysis 30 fragments of 100 points were selected. Afterwards, the relations between the initial perfusion parameters $\{x 1[i]=\operatorname{MPleft}[i]\}$ and $\{x 2[i]=\operatorname{MPright}[i]\}$ and their changes $\{\Delta x 1[i]\}$ and $\{\Delta x 2[i]\}, i=1,2 \ldots \ldots .100$ from the left and right sides were analyzed with multiple regression method. In order to do this, $a 0, a 1, a 2, b 0, b 1$, and $b 2$ parameters of regression equations were calculated for each subject:

$$
\left\{\begin{array}{l}
\Delta x 1=a 0+a 1 x 1+a 2 \times 2 \\
\Delta x 2=b 0+b 1 x 1+b 2 \times 2 .
\end{array}\right.
$$

Standard statistical tests were used to assess the significance of regression analysis results. For that purpose, standard errors of $s a 0, s a 1, s a 2, s b 0, s b 1$, and $s b 2$ regression coefficients were calculated; Fisher's F-test was applied to assess the validity of regression equations. The regression model underlay the mathematical model represented as a system of linear differential equations:

$$
\left\{\begin{array}{l}
d x 1 / d t=a 0+a 1 x 1+a 2 x 2 \\
d x 2 / d t=b 0+b 1 x 1+b 2 \times 2 .
\end{array}\right.
$$

Further searches for the solutions of this system and their stability were conducted with standard mathematical methods [7]. The calculations were performed in conditional perfusion units using Statistica and Excel for Windows (v. 6.0) software tools.

\section{RESULTS}

Mathematical model. At the first stage of the experiment the numerical values were calculated for the coefficients in multiple regression Eqs. (1). The calculations were performed in the initial state and following the hypoxic exposure. The results of regression analysis confirmed the relevance of regression coefficients and validity of selected regression model. The estimated numerical values of regression coefficients are presented in Table 1. The patterns of changes in MCR parameters described by the system of regression Eqs. (1) allowed presuming that the system of regression Eqs. (1) could be replaced by the analogous system of differential Eqs. (2) within subtle changes $\{\Delta x 1[i]\}$ and $\{\Delta x 2[i]\}$ that were indeed seen since the counting interval of periodogram was $\Delta t[i]=0.05 \mathrm{~s}$. We used such system of differential equations as the foundation for the mathematical model. It is known [7] that the solution of system of first-order differential equations with constant coefficients can be derived from the reduction of this system to a single linear differential equation of higher order. Therefore, the system (2) was reduced to a single second-order differential equation using simple algebraic manipulations:

$$
\begin{gathered}
d^{2} \times 2 / d t^{2}=(b 2+a 1) d x 2 / d t \\
+(b 1 a 2-a 1 b 2) x 2+(b 1 a 0-a 1 b 0), \\
\text { or }\left(T 1 p^{2}+T 2 p+1\right) \times 2=K,
\end{gathered}
$$

with $p$ designating the differential operator; $T 1, T 2$, and $K$ coefficients respectively equal to:

$$
\begin{gathered}
T 1=1 /(a 1 b 2-b 1 a 2), \\
T 2=(b 2+a 1) /(b 1 a 2-a 1 b 2), \\
K=(b 1 a 0-a 1 b 0) /(a 1 b 2-b 1 a 2) .
\end{gathered}
$$

The solution of equation (4) appeared as:

$$
x 2(t)=K+\exp (\alpha t)[C 1 \cos \beta t+C 2 \sin \beta t],
$$

where $C 1$ and $C 2$ constants were determined by the primary conditions $x 1(0)=A$ and $x 2(0)=B$, while $\alpha=$ $-T 2 / 2 T 1$ and $\beta=\sqrt{ }\left(4 T 1-T 2^{2}\right) / 2 T 1$ stood for the real and imaginary parts of the roots, respectively:

$$
\lambda_{1,2}=\left[-T 2 \pm \sqrt{ }\left(T 2^{2}-4 T 1\right)\right] / 2 T 1
$$

of the characteristic equation:

$$
\left(T 1 \lambda^{2}+T 2 \lambda+1\right) x 2=0 .
$$

We received the solution of the initial system of differential Eqs. (2) with regard to $x 2(t)$ variable describing the dynamic of bloodstream changes on the right $x 2(t)$. A similar solution could be obtained from the second equation of the initial differential equation system for $x 1(t)$ variable characterizing the dynamic of bloodstream changes on the left:

$$
x 1(t)=(d x 2 / d t-b 0-b 2 \times 2) / b 1 .
$$

Since the solution for $x 2(t)$ variable was already known and $d x 2 / d t$ could be easily attained by differentiating (8), equation (11) provided a comprehensive description of the dynamic in bloodstream changes of the left $\operatorname{arm} x 1(t)=\operatorname{MPleft}(t)$. Thus, a general solution $\{x 1(t), x 2(t)\}$ could be obtained for the system of linear differential Eqs. (2) characterizing the regulatory processes of MCR parameters in symmetrical areas of the paired human organs.

Stability of microcirculation. One of the important issues in the researches of physiological systems is the investigation of mechanisms providing the stability of their functioning. The results of this study demonstrated that the functioning of MCR system in symmetrical temporal head regions in humans followed 
MEZENTSEVA et al.

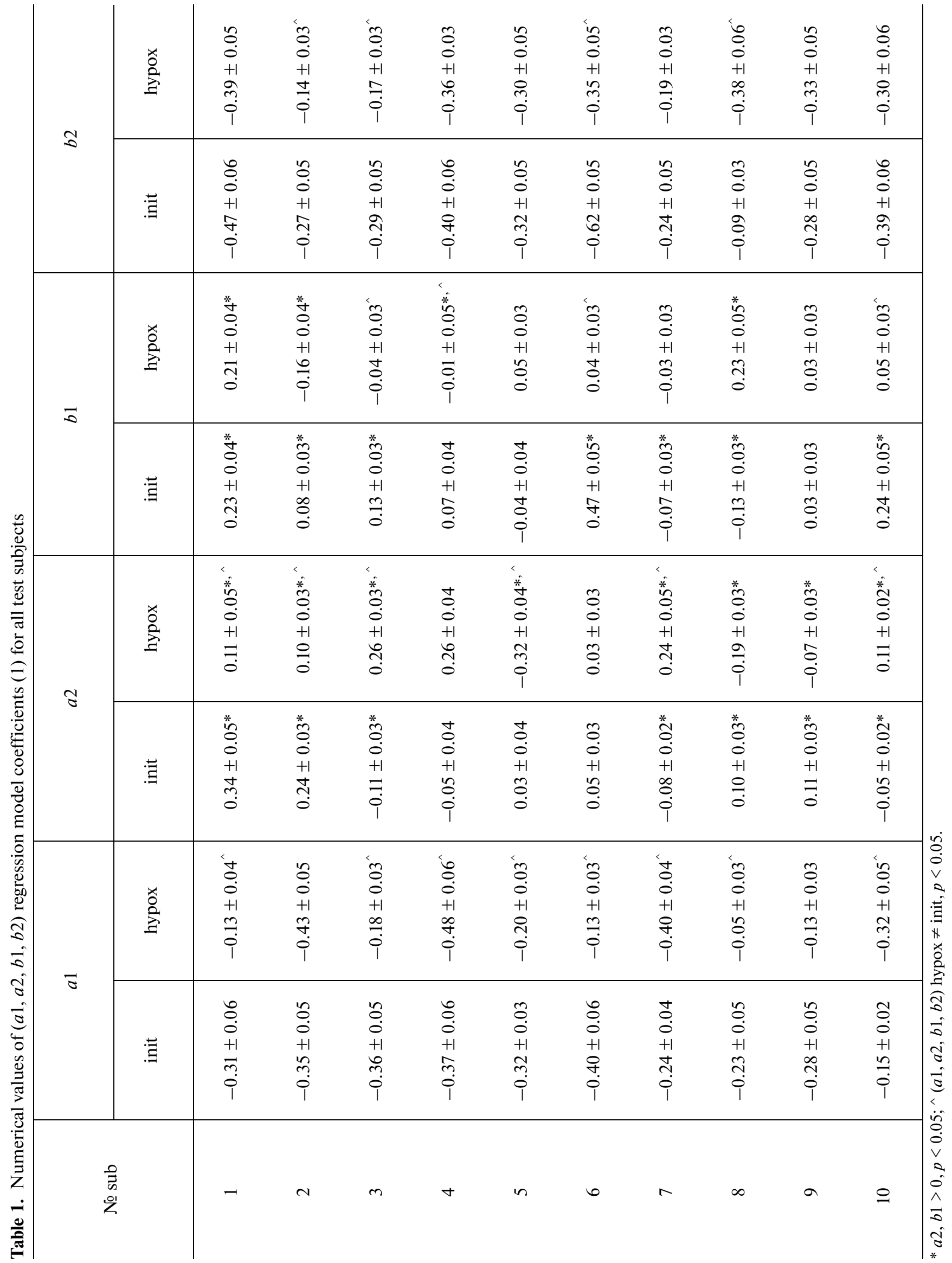



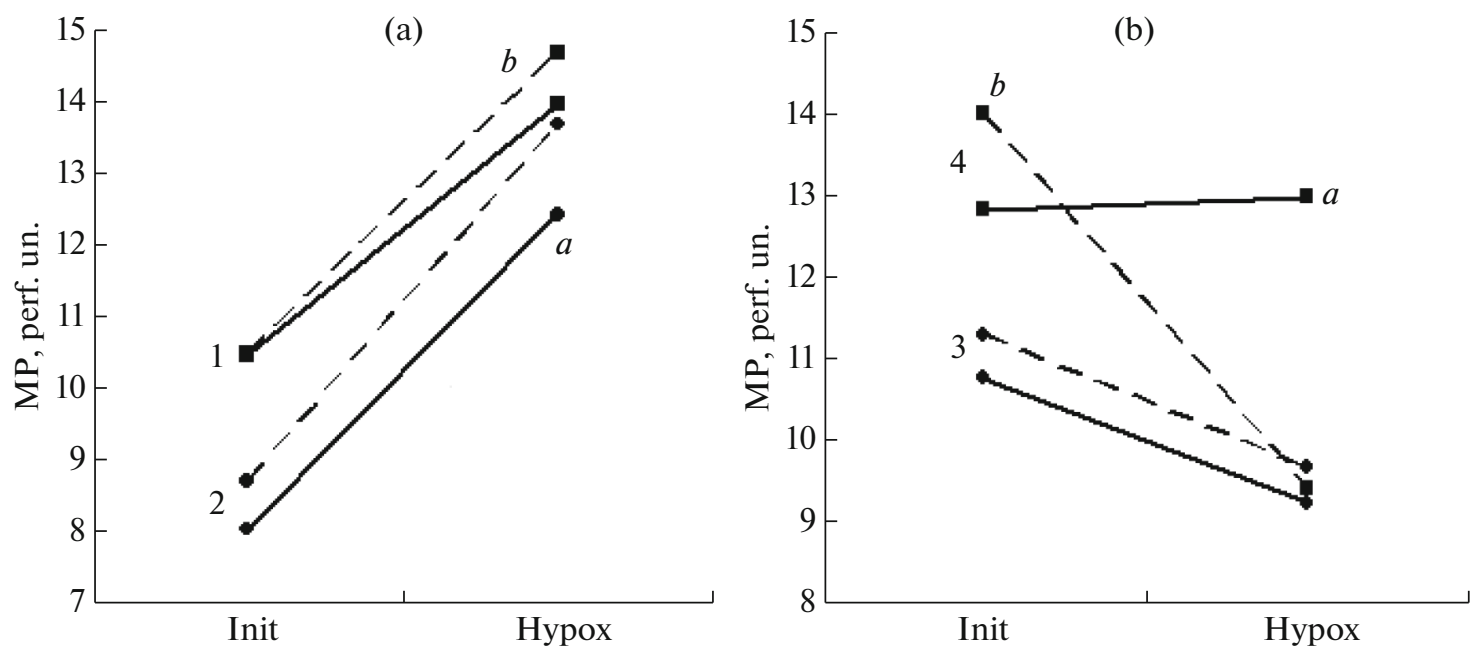

Fig. 1. The influence of short-term hypoxia on the perfusion parameters of left (MPleft) and right (MPright) temporal head regions in different subjects. (a) Subjects 1 and 2 with increasing MPleft and MPright after hypoxia; (b) subject 3 with decreasing MPleft and MPright after hypoxia, while in subject 4 MPright reduced dramatically and MPleft subtly elevated; $a$, changes in MPleft; $b$, changes in MPright.

strict mathematical laws described by a system of linear differential equations (2). Hence the stability conditions for this system could be investigated with the help of standard mathematical methods. According to the Routh-Hurwitz stability criterion [7], the stability test for the system of linear differential equations of $n$ order confines to the identification of negativity conditions for the real parts of corresponding characteristic equation. In our case, all roots of the characteristic Eq. (10) were negative and the system of differential Eqs. (2) would have stable solutions if:

$$
\begin{gathered}
T 1=1 /(a 1 b 2-b 1 a 2)>0, \\
T 2=-(b 2+a 1) /(a 1 b 2-b 1 a 2)>0,
\end{gathered}
$$

and variables were bound by a condition:

$$
\zeta=T 2 / 2 T 1<1 \text {. }
$$

This condition meant that the roots of characteristic Eq. (10) were complex. Because the differential Eq. (3) described an oscillatory process, which characteristics depended on specific numerical values of $a 0, a 1, a 2, b 0, b 1$, and $b 2$ parameters, different variants of the behavior of studied oscillatory system were possible depending on $\zeta$ value. The oscillability of the conversion decreases with the growth of $\zeta$, decaying to zero by $\zeta=1$.

Influence of hypoxia on the stability of microcirculation. The study results showed that hypoxic influences induced the changes in MCR parameters in both right and left observation sides, these changes being remarkable for strongly pronounced left-right asymmetry and individual for different subjects. In $80 \%$ of volunteers hypoxia provoked the increase in the perfusion parameters on the left side (MPleft) and the decrease in the rest $20 \%$. Moreover, the perfusion parameters on the right (MPright) elevated in $50 \%$ of subjects under the influence of hypoxia and reduced in the other $50 \%$.

Figure 1 shows the examples of different types of reaction of perfusion parameters on short-term hypoxia. Simultaneous elevation of MPleft and MPright was observed in the subjects in Fig. 1a exposed to short-term hypoxia, whereas in one subject from Fig. 1b MPleft and MPright simultaneously decreased and in another one MPright reduced dramatically and MPleft increased slightly. As a result, the response to short-term hypoxic conditions was characterized by high individual variability. It should be emphasized that the decline in perfusion parameters under the influence of short-term hypoxia was detected in smokers. No individual features of the reactions in each subject could be determined after averaging across all subjects. The mean initial perfusion value on the left was MPleft(init) $=9.44 \pm$ 0.54 perf. un., after hypoxia rising to MPleft(hypox) $=$ $10.55 \pm 0.60$ perf. un.; the initial mean perfusion value on the right was MPright(init) $=9.91 \pm 0.75$ perf. un . and after hypoxia MPright(hypox) $=10.67 \pm$ 0.69 perf. un., with no statistical changes.

The next experimental stage was aimed to investigate the quantitative patterns in the fluctuations of MCR parameters using multiple regression analysis by assessing $a 0, a 1, a 2, b 0, b 1$, and $b 2$ coefficients of regression model (1). The calculation results are presented in Table 1. As demonstrated in Table 1, the estimated values of $a 2$ regression coefficients statistically differed from zero $(p<0.05)$ in the majority of subjects. This indicated that perfusion changes on the left $(\Delta x 1=\Delta$ MPleft $)$ depended from both the initial value of this parameter on the left $(x 1=$ MPleft $)$ and on the right $(x 2=$ MPright). Similarly, on the right observa- 

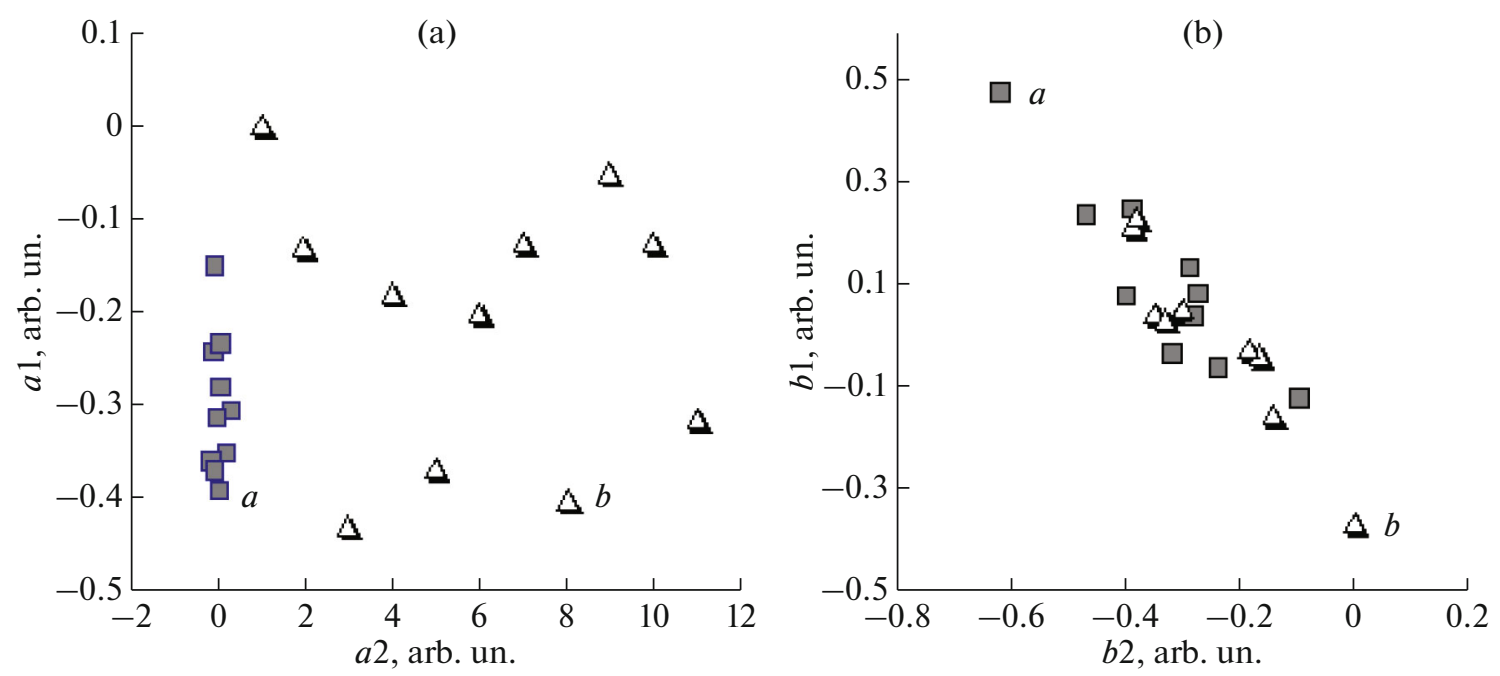

Fig. 2. The changes in the location of geometric points in the phase plane of regression coefficients after short-term hypoxic influence: (a) on the left observation side $(a 1, a 2)$; (b) on the left observation side $(b 1, b 2) ; a$, initial state; $b$, state after hypoxic exposure.

tion side the estimated values of $b 1$ regression coefficients statistically differed from zero $(p<0.05)$. This proved that perfusion changes on the right side $(\Delta x 2=$ $\Delta$ MPright) depended from both the initial value of this parameter on the right $(x 2=$ MPright $)$ and on the left $(x 1=$ MPleft $)$. Therefore, the results of regression analysis suggested that the changes in amplitudes of microcirculatory fluctuations in symmetrical temporal head regions depended on the initial values of these parameters both ipsi- and contralaterally.

The results of regression analysis discovered the most pronounced influence of short-term hypoxia on the regression coefficients of the left side compared to the right one. This is illustrated in Fig. 2, showing the changes in the locations of geometric points in the phase plane of regression coefficients of the left (Fig. 1a) and right (Fig. 2b) observation sides. The presence of changes in numerical values of regression coefficients under the influence of short-term hypoxia can be also seen in Table 1, referring to which the most expressed influence of short-term hypoxia was exerted on the coefficients on the left side of observation. Significant changes in the $a 1$ and $a 2$ coefficients were registered in $80 \%$ of subjects, in contrast with $40 \%$ of cases for $b 1$ and $b 2$ coefficients on the right side. It could be deduced that short-term exposure to hypoxia in MCR system affected the balance between the distribution of blood supply in right and left observation sides. This supports the assumption that left-right asymmetry of MCR parameters plays an important role in the mechanisms of autoregulation of regional circulation and the balance between regulatory effects of symmetrical regions in MCR system redistributes under external influences in order to maintain stable functioning of the integrated MCR system.
The following step of the trial involved stability test of MCR system. For this purpose, numerical values of $T 1, T 2$, and $\zeta$ stability parameters were calculated and the fulfillment of conditions (13)-(15) was verified. The test results are presented in Table 2, demonstrating that the values of regression coefficients both in the initial state and immediately after short-term hypoxic exposure of all subjects satisfied the stability requirements; no unstable states were found. Stability test results discovered that personalized assessment of stability degree in short-term hypoxic conditions was influenced by the degree of sustainability of microcirculation between the left and right sides, rather than by mean values of perfusion parameters from the left and right, as seen in the elaborated objective stability parameters $(T 1, T 2$, and $\zeta)$. For instance, the perfusion parameter on the right decreased in subject 2 after the exposure to short-term hypoxia (MPright(init) = 14.03; MPright(hypox) = 9.42); however, this did not suggest the reduction of MCR system stability of that subject. On the contrary, $T 1, T 2$, and $\zeta$ stability parameters elevated in that subject after a short-term exposure to hypoxia, indicating a positive influence on MCR system stability. A similar increase in $T 1, T 2$, and $\zeta$ stability markers with the decrease in perfusion parameters was observed in subjects 7 and 8, suggesting that the personalized assessment of resistance to short-term hypoxic exposure was affected by the sustainability degree of the microcirculation between right and left sides represented by the values of $T 1, T 2$, and $\zeta$ stability markers, rather than by the mean values of perfusion parameters from the left and right sides. The results of statistical analysis for changes in $T 1, T 2$, and $\zeta$ stability markers showed that hypoxic influence was overall tending to enhance the stability of MCR system, as seen from Fig. 3. Figure 3 shows the mean 
Table 2. Numerical values of $\{T 1, T 2, \zeta\}$ parameters determining the stability of mathematical model (2) in the initial state and after short-term hypoxic exposure, as well as the results of stability test of the MCR system (TRUE or FALSE) for all subjects

\begin{tabular}{c|c|c|c|c|c|c|c|c|c}
\hline \multirow{2}{*}{ № sub } & \multicolumn{3}{|c|}{$T 1$} & \multicolumn{3}{c|}{$T 2$} & \multicolumn{3}{c}{$\zeta$} \\
\cline { 2 - 9 } & init & hypox & T1>0 & init & hypox & T2>0 & init & hypox & $\zeta<1$ \\
\hline 1 & 14.95 & 37.47 & TRUE & 11.64 & 19.40 & TRUE & 0.39 & 0.26 & $T R U E$ \\
2 & 12.78 & 12.83 & TRUE & 8.01 & 7.38 & TRUE & 0.31 & 0.29 & TRUE \\
3 & 8.54 & 24.57 & TRUE & 5.54 & 8.55 & TRUE & 0.32 & 0.17 & TRUE \\
4 & 6.58 & 5.67 & TRUE & 5.08 & 4.76 & TRUE & 0.39 & 0.42 & $T R U E$ \\
5 & 9.82 & 13.25 & TRUE & 6.23 & 6.68 & TRUE & 0.32 & 0.25 & TRUE \\
6 & 4.49 & 23.16 & TRUE & 4.55 & 11.06 & TRUE & 0.51 & 0.24 & TRUE \\
7 & 19.19 & 12.08 & TRUE & 9.22 & 7.13 & TRUE & 0.24 & 0.30 & TRUE \\
8 & 28.69 & 16.09 & TRUE & 9.41 & 6.93 & TRUE & 0.16 & 0.22 & TRUE \\
9 & 13.25 & 22.80 & TRUE & 7.45 & 10.43 & TRUE & 0.28 & 0.23 & TRUE \\
10 & 13.98 & 11.19 & TRUE & 7.56 & 6.88 & TRUE & 0.27 & 0.31 & TRUE \\
\hline
\end{tabular}

values of $T 1$ and $T 2$ stability markers and $\zeta$ parameter, characterizing the expressiveness of oscillability of fluctuations in the microcirculation of MCR system for all 10 volunteers (Fig. 3a). Figure 3 also demonstrates that the numerical values of $T 1$ and $T 2$ stability markers throughout the group averagely increased together with the elevation of expressiveness of oscillability of fluctuations in the microcirculation $\zeta$ under the influence of short-term hypoxia.

\section{DISCUSSION}

The problems of stability of the circulation response to hypoxic loads are discussed in numerous studies due to the wide application of hypoxic trainings in the sports medicine and in nonpharmacological prevention and rehabilitation of patients suffering from different diseases [8-11]. Nevertheless, all of these studies are narrative, because of the absent consideration of mathematical regulations of right-left hemodynamic balance in the system of regional circulation that we have discovered in the present investigation. The novel computational and experimental method was introduced in this study for the assessment of MCR system resistance to hypoxic exposure based on the analysis of solutions for differential equation system describing the functioning of right-left hemodynamic balance in the system of regional circulation. The experiments discovered that hypoxic influences altered the numerical values of the stability parameters in all subjects within the boundaries of stability levels. However, the reaction to hypoxic influences was individual for different subjects. This corresponds with the results of our previous researches [8],
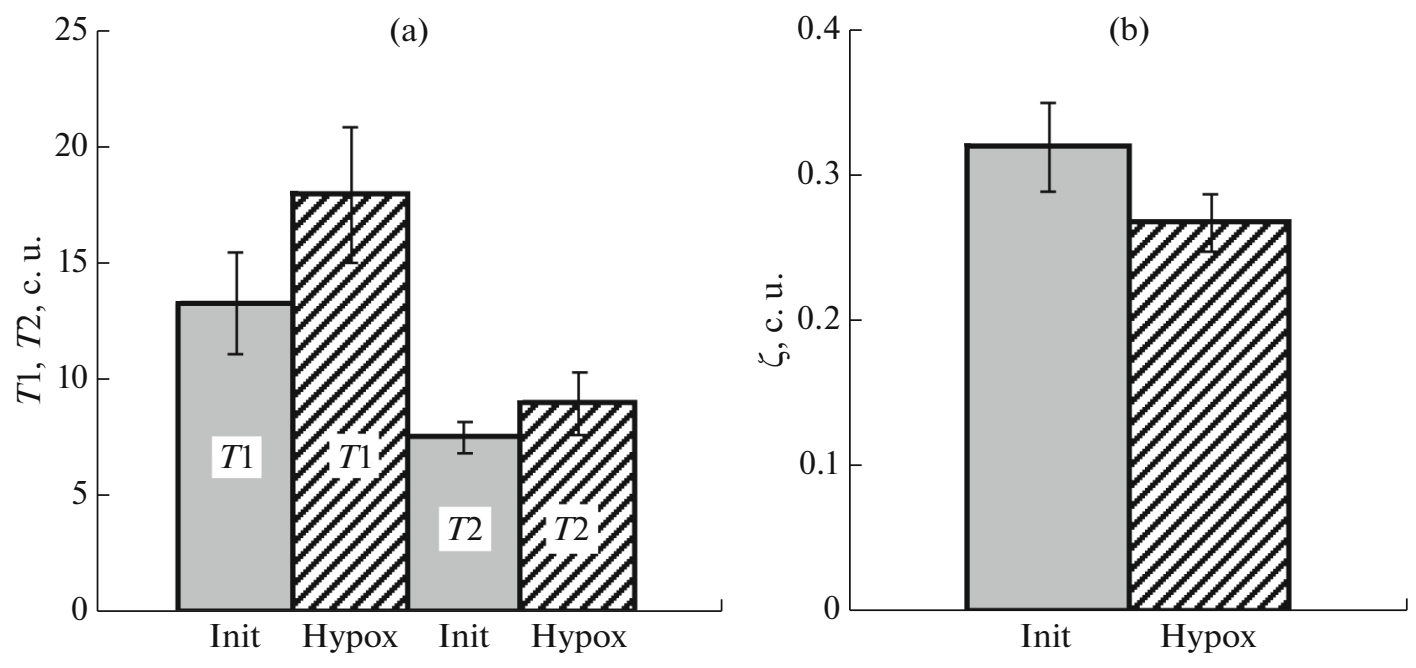

Fig. 3. Mean values of (a) $T 1$ and $T 2$ and (b) $\zeta$ stability markers in the initial state and after short-term hypoxic exposure. 
showing that short-term hypoxic influence could induce substantial response of the microcirculatory bloodstream. The endothelial and neurogenic vasculomotor components of the microhemodynamic regulation were enhanced in the volunteers, the type of microcirculatory response depending on the baseline sensitivity of subjects to hypoxia and was individual for different subjects. In the present study we have not only confirmed the results of these researches, but also explained the reasons for high individual variability of the reactions of MCR parameters to hypoxia. We demonstrated that the functioning of MCR system was described by strict mathematical equations defining the sustainability of circulation of right and left microcirculatory bloodstreams devoted to maintain the stability of the whole system. Hence, the response to hypoxia in some subjects was characterized by elevated perfusion on one or another observation side, and was reduced in other subjects. The right-left balance of circulation in symmetrical regions was the pivotal factor rather than the perfusion rate on one or another observation side. The degree of sustainability of circulation in symmetrical regions of microcirculatory bloodstream was determined by the parameters of mathematical model described by the system of differential Eqs. (2). The introduced criteria of correlation between the numerical values of these parameters expressed in Eqs. (13)-(15) determined the stability conditions for the functioning of integrated MCR system. This system functions so that any external disturbances provoke a compensatory reaction aimed to maintain the system stability. The research results showed that hypoxic influences led to different changes in the perfusion parameters from the left and right sides in different subjects, though the expressiveness and directionality of systemic compensatory reaction to hypoxia was the same for all subjects. This reaction tended to increase the numerical values of $T 1$ and $T 2$ parameters determining the system stability. Therefore, hypoxic influences induced the compensatory reaction aiming to maintain the stable functioning of integrated circulatory system in symmetrical organs. The results of present studies confirmed the principle of right-left hemodynamic balance earlier introduced by Nogina [12], defining the spatiotemporal organization of hemodynamic system and eliciting its integrated role regarding the supplied organs. The maintenance of right-left hemodynamic balance is the crucial life-supporting factor for stability of integrated hemodynamic system and involves all hierarchy of autonomic and central regulation of the vascular tone.

\section{CONCLUSIONS}

(1) It was found that the changes in fluctuation amplitude of microcirculation in symmetrical temporal head regions depended on the initial values of these parameters both from the ipsi- and contralateral sides of observation.
(2) It was shown that the changes in perfusion of symmetrical temporal head regions were always negatively correlated with instantaneous perfusion values ipsilaterally from the observation side, while both positive and negative correlations could be found on the contralateral side.

(3) Regression equations were derived, describing the correlations between the synchronous changes in MCR parameters of symmetrical temporal head regions and their initial values on the left and right sides of observation. It was demonstrated that the numerical values of $a 1, a 2, b 1$, and $b 2$ parameters of regression equations could be used to estimate the stability of MCR system functioning.

(4) The individual assessments of numerical values for the parameters of regression equations ( $a 1, a 2, b 1$, and $b 2$ ) were obtained for each subject in the initial state and after short-term hypoxic exposure, allowing to estimate the resistance of MCR system to shortterm hypoxic influences.

(5) It was discovered that short-term hypoxic exposure affected the numerical values of the stability parameters in all subjects within the boundaries of stability levels, however the reaction to hypoxia was very individual for different subjects.

(6) Introduced method could be used in personalized assessments of fitness levels and resistance to hypoxia in different groups of subjects.

(7) Introduced method allowed determining the quantitative criteria distinguishing between the normal stable and pathological unstable modes of MCR system functioning and could be applied in practical medicine to evaluate the state of MCR system in patients and prognosticate the probability of its transition in the pathological mode.

\section{FUNDING}

The study was financially supported by the Federal Budget of the Russian Federation within the State task of Anokhin Research Institute of Normal Physiology and Sklifosovsky Institute of Clinical Medicine, Sechenov First Moscow State Medical University (Moscow, Russia).

\section{COMPLIANCE WITH ETHICAL STANDARDS}

All procedures performed in studies involving human participants were in accordance with the ethical standards, the 1964 Helsinki Declaration and its later amendments, and approved by the ocal bioethical committee of the Anokhin Research Institute of Normal Physiology (Moscow, Russia). Informed consent was obtained from all individual participants involved in the study.

\section{CONFLICT OF INTEREST}

The authors declare that they have no conflicts of interest.

HUMAN PHYSIOLOGY Vol. $48 \quad$ No. 12022 


\section{OPEN ACCESS}

This article is licensed under a Creative Commons Attribution 4.0 International License, which permits use, sharing, adaptation, distribution and reproduction in any medium or format, as long as you give appropriate credit to the original author(s) and the source, provide a link to the Creative Commons license, and indicate if changes were made. The images or other third party material in this article are included in the article's Creative Commons license, unless indicated otherwise in a credit line to the material. If material is not included in the article's Creative Commons license and your intended use is not permitted by statutory regulation or exceeds the permitted use, you will need to obtain permission directly from the copyright holder. To view a copy of this license, visit http://creativecommons.org/licenses/by/4.0/.

\section{REFERENCES}

1. Mikhailichenko, L.A., Microcirculation indicators of paired organs and tissues of experimental healthy animals, Reg. Krovoobrashch. Mikrotsirkulyatsiya, 2007, vol. 6 , no. 1, p. 164.

2. Mezentseva L.V. and Pertsov S.S., Synchronous changes in microcirculation parameters of the upper limbs in asymmetric physical loads, Hum. Physiol., 2020, vol. 46 , no. 6 , p. 671 .

3. Ladozhskaya-Gapeenko, E.E. and Khrapov, K.N., Possibilities of laser-Doppler flowmetry in assessment of functional state of microcirculation, Reg. Krovoobrashch. Mikrotsirkulyatsiya, 2020, vol. 19, no. 3, p. 39.

4. Fedorovich, A.A., Microcirculation of the human skin as a study object, Reg. Krovoobrashch. Mikrotsirkulyatsiya, 2017, vol. 16, no. 4, p. 11.
5. Martini, R. and Bagno, A., The wavelet analysis for the assessment of microvascular function with the laser Doppler fluxmetry over the last 20 years. Looking for hidden informations, Clin. Hemorheol. Microcirc., 2018, vol. 70, no. 2, p. 213.

6. Low, D.A., Jones, H., Cable, N.T., et al., Historical reviews of the assessment of human cardiovascular function: interrogation and understanding of the control of skin blood flow, Eur. J. Appl. Physiol., 2020, vol. 120, no. 3 , p. 1 .

7. El'sgol'ts, L.E., Differentsial'nye uravneniya $i$ variatsionnoe ischislenie (Differential Equations and Variation Calculation), Moscow: Nauka, 1969.

8. Glazachev, O.S. and Dudnik, E.N., Microcirculatory reactivity features in apparently healthy individuals during acute moderate hypoxia and hyperoxia modeling, Hum. Physiol., 2013, vol. 39, no. 4, p. 400.

9. Zelenkova, I.E., Zotkin, S.V., Korneev, P.V., et al., Variability of hypoxic resistance of athletes of various qualifications and sports specialization, Sport. Med.: Nauka Prakt., 2016, vol. 6, no. 4, p. 5.

10. Maleev, D.O., Determination of the individual resistance of the organism of highly qualified skiers-racers to acute hypoxia, Chel., Sport., Med., 2015, vol. 15, no. 4, p. 19.

11. Mateika, J.H., El-Chami, M., Shaheen, D., and Ivers, B., Intermittent hypoxia: a low-risk research tool with therapeutic value in humans, J. Appl. Physiol., 2015, vol. 118, no. 5, p. 520.

12. Mezentseva, L.V., Fundamental principles of system organization of cerebral circulation (according to the owrks of S.P. Nogina), Akad. Zh. Zap. Sib., 2018, vol. 14 , no. $6(77)$, p. 4.

Translated by E. Sherstyuk 